\title{
Cluster growth in two- and three-dimensional granular gases
}

\author{
S. Miller ${ }^{1}$ and S. Luding ${ }^{1,2, *}$ \\ ${ }^{1}$ Institut für Computeranwendnungen 1, Universität Stuttgart, Pfaffenwaldring 27, D-70569 Stuttgart, Germany \\ ${ }^{2}$ Particle Technology, DelftChemTech, TU Delft, Julianalaan 136, 2628 BL Delft, The Netherlands \\ (Received 28 April 2003; revised manuscript received 18 August 2003; published 30 March 2004)
}

\begin{abstract}
Dissipation in granular media leads to interesting phenomena such as cluster formation and crystallization in nonequilibrium dynamical states. The freely cooling system is examined concerning the energy decay and the cluster evolution in time, both in two and three dimensions. We also suggest an interpretation of the threedimensional cluster growth in terms of percolation theory, but this point deserves further study.
\end{abstract}

DOI: 10.1103/PhysRevE.69.031305 PACS number(s): 45.70.Qj, 45.70.-n, 64.60.Ak, 81.05.Rm

\section{INTRODUCTION}

Granular media are interesting multiparticle systems with a rich phenomenology [1-5]. They can form a hybrid state between a fluid and a solid, where the behavior is controlled by the balance between energy input and energy dissipation. Energy input leads to a reduction of the density due to more collisions and increasing pressure, so that the material can flow. In the absence of energy input, e.g., in freely cooling systems, granular materials become denser, i.e., they locally "solidify" due to dissipation. Because of mass conservation, the local densification is accompanied by a density decrease in other parts of the system, giving rise to complex patterns and structures, with an interesting time evolution. However, theoretical approaches are nonclassical and appear often extremely difficult, so there is still active research directed towards the better understanding of granular media.

The subject of this paper is the pattern formation via clustering in a dissipative, freely cooling granular gas [6-12]. The basic idea of clustering is that in an initially homogeneous freely cooling granular gas, fluctuations in density, velocity, and temperature cause a position dependent energy loss. Due to strong locally inhomogeneous dissipation, pressure and energy drop rapidly and material moves from "hot" to "cold" regions, leading to even stronger dissipation and thus causing the density instability with ever growing clusters until eventually, clusters reach system size (see Fig. 1).

We investigate this clustering instability with respect to different dissipation rates and different system sizes. Even though many of our findings are empirical, we attempt to reduce the complexity of the evolution of the system by use of simple scaling laws.

In Sec. II, we explain in detail the simulation approach. The results of our numerical experiments are discussed in Sec. III. Finally, in Sec. IV a summary and a discussion are given.

\section{SIMULATION DETAILS}

A granular gas can be idealized as an ensemble of hard spheres in which the energy loss that accompanies the collision of macroscopic particles is modeled with a single coef-

*Email address: S.Luding@tnw.tudelft.nl ficient of restitution $r$. In the simplest case the particles are identical in size and mass and there are no interparticle forces between collisions.

Details about initial and boundary conditions are given in Sec. II A. The microscopic dynamics of the motion and the collision of the particles is discussed in Sec. II B and the simulation method is explained in Sec. II C. Section II D deals with the inelastic collapse, a problematic artifact of the hard sphere model with dissipation.

\section{A. Initial and boundary conditions}

The simulation volume consists of a box with equal side length and periodic boundary conditions in two or three dimensions.

An initial state with random particle positions and velocities is prepared in the following way: The particles first sit on a regular lattice and have a Maxwellian velocity distribution with a total momentum of zero. Then the simulation is started without dissipation and runs for about $10^{2}$ collisions per particle. This state is now used as initial configuration for the dissipative simulations.

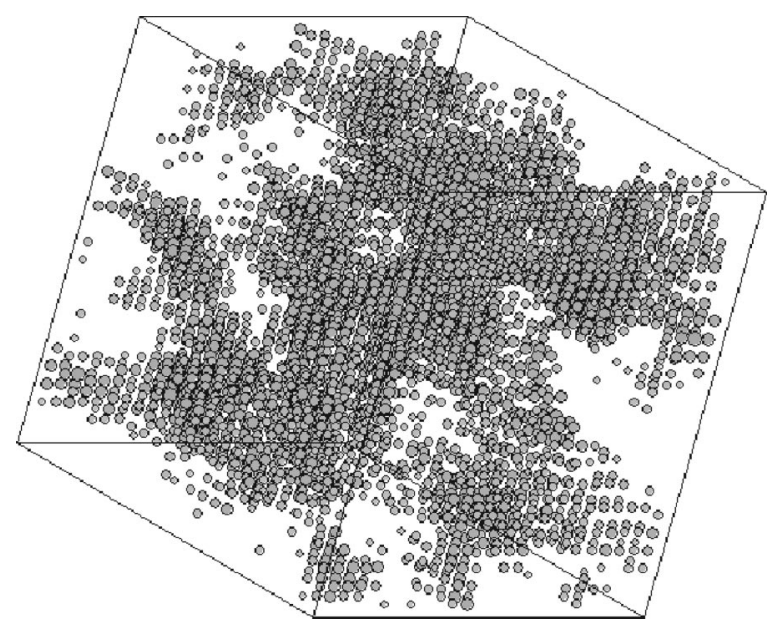

FIG. 1. Density distribution in a snapshot of a 3D system with 512000 particles, volume fraction $\rho=0.25$, and restitution coefficient $r=0.3$. Each sphere represents a cell with about 40 particles in average. The size of the spheres is proportional to the local density; very small spheres (corresponding to low densities) are omitted. 


\section{B. Microscopic dynamics}

Between collisions no forces act upon the particles and they move at constant velocity. The particles are idealized as hard spheres. This means that collisions take infinitesimal time and involve only two particles. Conservation of momentum leads to the collision rule $[6,13]$

$$
\mathbf{v}_{1 / 2}^{\prime}=\mathbf{v}_{1 / 2} \mp \frac{1+r}{2}\left[\hat{\mathbf{k}} \cdot\left(\mathbf{v}_{1}-\mathbf{v}_{2}\right)\right] \hat{\mathbf{k}}
$$

where a prime indicates the velocities $\mathbf{v}$ after the collision, $\hat{\mathbf{k}}$ is a unit vector pointing along the line of centers from particle 1 to particle 2, and $r$ is the coefficient of restitution. The relative tangential velocity does not change during a collision, the relative normal velocity changes its sign and is reduced by a factor $1-r$. Energy dissipation is proportional to $\lambda=1-r^{2}$, so that the elastic limit $r=1$ implies $\lambda=0$, i.e., no dissipation, while $r<1$ implies $\lambda>0$.

\section{Event-driven molecular dynamics}

The simulation of hard spheres can be handled efficiently with event-driven molecular dynamics $[14,15]$. The collisions are the events which have to be treated by the algorithm. Between these collisions the particles move on trivial trajectories and so the algorithm can easily compute the point of time $t_{12}$ of the next collision of two particles 1 and 2 as

$$
t_{12}=t_{0}-\mathbf{r}_{12} \cdot \mathbf{v}_{12} / v_{12}^{2}+\sqrt{\left(\mathbf{r}_{12} \cdot \mathbf{v}_{12}\right)^{2}-\left(r_{12}^{2}-d^{2}\right) v_{12}^{2}} / v_{12}^{2},
$$

where $\mathbf{v}_{12}=\mathbf{v}_{2}\left(t_{0}\right)-\mathbf{v}_{1}\left(t_{0}\right)$ and $\mathbf{r}_{12}=\mathbf{r}_{2}\left(t_{0}\right)-\mathbf{r}_{1}\left(t_{0}\right)$ are the relative velocities and positions of the particles at time $t_{0}$, and $d$ is the diameter of a particle.

The algorithm processes the events one after another. After a collision the positions and velocities of the two involved particles are updated; the state of all other particles remains unchanged. For the two colliding particles, new events are calculated and the next future event is stored in the event priority queue for both particles. The next event is obtained from the priority queue, the new positions and velocities after the collision for the collision partners are updated, and so on. Neighborhood search is enhanced with standard linked cell methods [16], where the cell change of a particle is treated as a new event type. The details of the algorithm can be found in Refs. [14,15,17].

\section{Avoiding the inelastic collapse with the time cutoff model}

Our model makes use of hard spheres with an infinitely stiff interaction potential. This is an idealization of real physical particles and can lead to the dramatic consequence of inelastic collapse: An infinite number of collisions occurs in finite time. This singularity is unphysical, of course, and a major drawback for numerical simulations, too. But it has been shown that one can circumvent this artifact of the model in the following way [18]: If two consecutive collisions of a particle happen within a small time $t_{c}$, dissipation
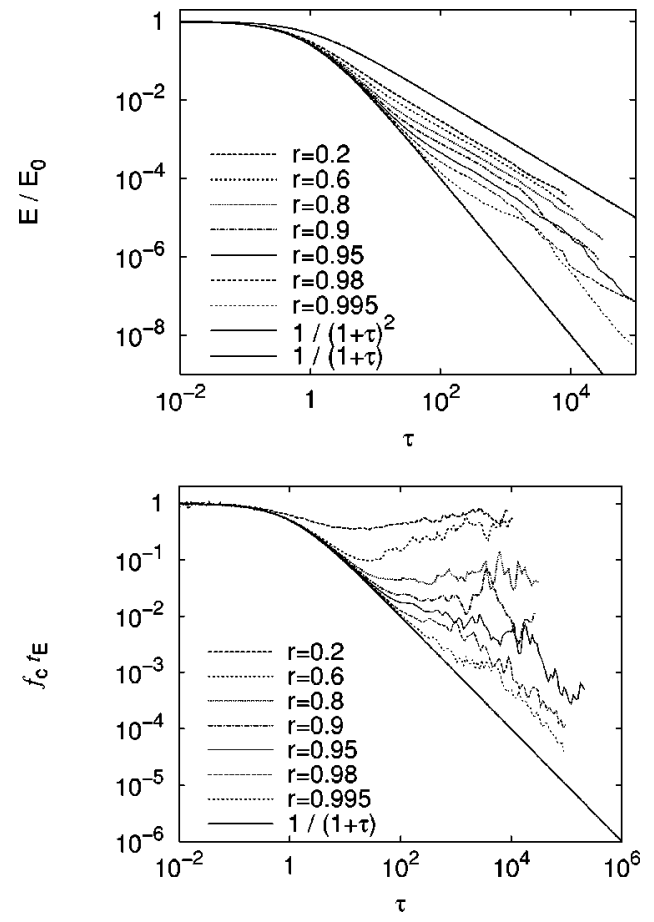

FIG. 2. Decay of the kinetic energy $E$ (top) and the collision frequency $f_{c}$ (bottom) plotted against scaled time $\tau$ in a 2D system with $N=316^{2}=99856$ particles, volume fraction $\rho=0.25$, and different restitution coefficients $r$ (increasing $r$ from top to bottom). The thick solid lines correspond to the theoretical predictions as given in the inset.

is switched off for the second collision. This time step $t_{c}$ corresponds to the duration of the contact of physical particles.

There exist other deterministic and random models which prevent inelastic collapse. Even though many of them lack a solid theoretical background and physical motivation, their details should be insignificant for the physical evolution of the system anyway, since only a small negligible fraction of the particles in the system is involved in the inelastic collapse. For an extensive discussion see Ref. [18].

\section{NUMERICAL EXPERIMENTS}

The simulation is started from a homogeneous system, prepared as described in Sec. II A. Depending on the dissipation $\lambda$, the density $\rho$, and the number $N$ of particles, the system remains in the homogeneous cooling state (HCS) for some time, until clustering starts and the system becomes inhomogeneous.

We discuss the evolution of the kinetic energy and the collision frequency of the system in Sec. III A. In Sec. III B we investigate the clustering by means of appropriate measures of the cluster size distribution. In Secs. III C and III D we encounter interesting parallels with percolation theory and discuss several critical exponents.

\section{A. Kinetic energy and collision frequency}

Dissipative collisions lead to a decay of the kinetic energy and the collision frequency (see Figs. 2 and 3). From these 

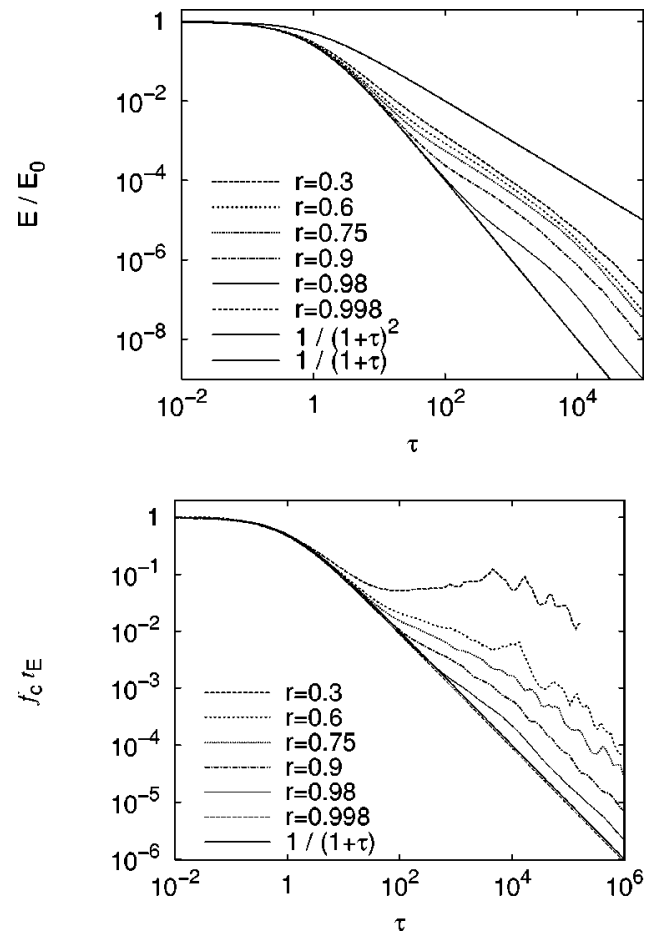

FIG. 3. Decay of the kinetic energy $E$ (top) and the collision frequency $f_{c}$ (bottom) plotted against scaled time $\tau$ in a 3D system with $N=80^{3}=512000$ particles, volume fraction $\rho=0.25$, and different restitution coefficients $r$ (increasing $r$ from top to bottom). The thick solid lines correspond to the theoretical predictions as given in the inset.

figures three different regimes can be clearly distinguished. They are as follows.

(1) The HCS at the beginning, when no clusters have formed yet, is well understood $[19,20]$. The decay of the kinetic energy $E$ is governed by the equation

$$
E(\tau)=\frac{E(0)}{(1+\tau)^{2}},
$$

with the scaled time $\tau=(\lambda / 2 D)\left(t / t_{E}\right) . D$ is the dimension of the system and $t_{E}$ is the initial Enskog collision time $t_{E}$ $=(\sqrt{\pi} d) /\left[2 \sqrt{D} v \rho g_{d}(\rho)\right] . v$ is the mean velocity of a particle, $d$ its diameter, $\rho$ is the volume fraction, and $g_{d}$ is the contact probability. In two dimensions (2D) $g_{d}(\rho)=(1$ $-7 \rho / 16) /(1-\rho)^{2}$ and in 3D $g_{d}(\rho)=(1-\rho / 2) /(1-\rho)^{3}$.

The evolution of the collision frequency per particle $f_{c}(\tau)$ with time is given by

$$
f_{c}(\tau)=t_{E}^{-1}(0) \sqrt{\frac{E(\tau)}{E(0)}}
$$

and it is the natural time scale controlling the evolution of the system in the HCS.

(2) When clusters start to grow, the decays deviate from these laws. In the cluster-growth regime, the decay of the kinetic energy slows down. Furthermore, the collision frequency fluctuates a lot and can even increase. Note that the collision frequency is no longer a natural time scale of the
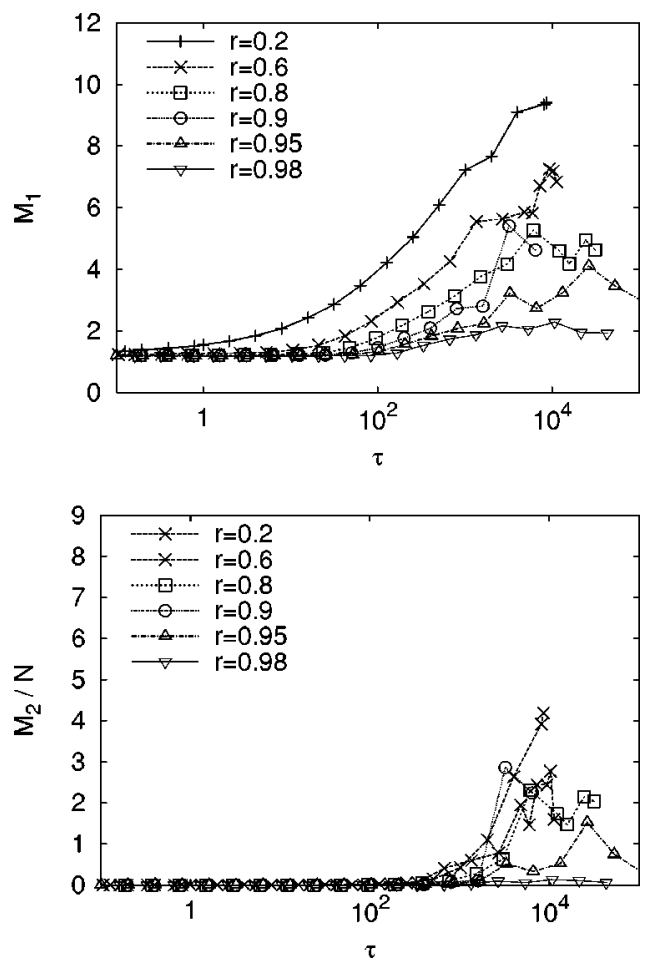

FIG. 4. Growth of the first moment $M_{1}$ (top) and the second moment $M_{2}$ (bottom) of the cluster size distribution plotted against scaled time $\tau$ in a $2 \mathrm{D}$ system with $N=99856$ particles, volume fraction $\rho=0.25$, and different restitution coefficients $r$ (increasing $r$ from top to bottom).

system, since it is mainly determined by cluster-cluster collisions, where it increases strongly. The deviation from Eq. (3) occurs earlier and is more dramatic for larger dissipation $\lambda$, i.e., smaller $r$. However, the cluster-growth regime is characterized by an energy decay around $E \sim \tau^{-1}$, independently of $r$, cf. Ref. [10]. (In 3D the exponent might be slightly larger than unity; a best fit yields $E \sim \tau^{-1.1 \pm 0.1}$.) In contrast, the evolution of the collision frequency with time depends on $r$ during cluster growth. This regime is more distinct for large dissipation $\lambda$ and large system sizes $N^{1 / D}$ and can, e.g., barely be seen for $r=0.98$ in Fig. 3 .

(3) Finally, when the largest cluster in the population has reached system size in the saturation regime, the cooling resembles the homogeneous cooling state in so far that $E(\tau) \propto \tau^{-2}$ and $f_{c}(\tau) \propto \tau^{-1}$, even if the latter still shows large fluctuations. In Fig. 2 this regime is not clearly visible, because the simulation times are not long enough for this system.

More details of the 2D situation were discussed in Refs. $[8,18]$ and references therein. Further studies on the threedimensional systems are in progress.

\section{B. Cluster growth}

The energy loss of the particles first leads to a reduced separation velocity after collision and eventually to the formation of clusters. But the definition of a cluster suffers from the fact that it takes an infinite number of collisions for the particles to stay in permanent contact with each other [9]. So 

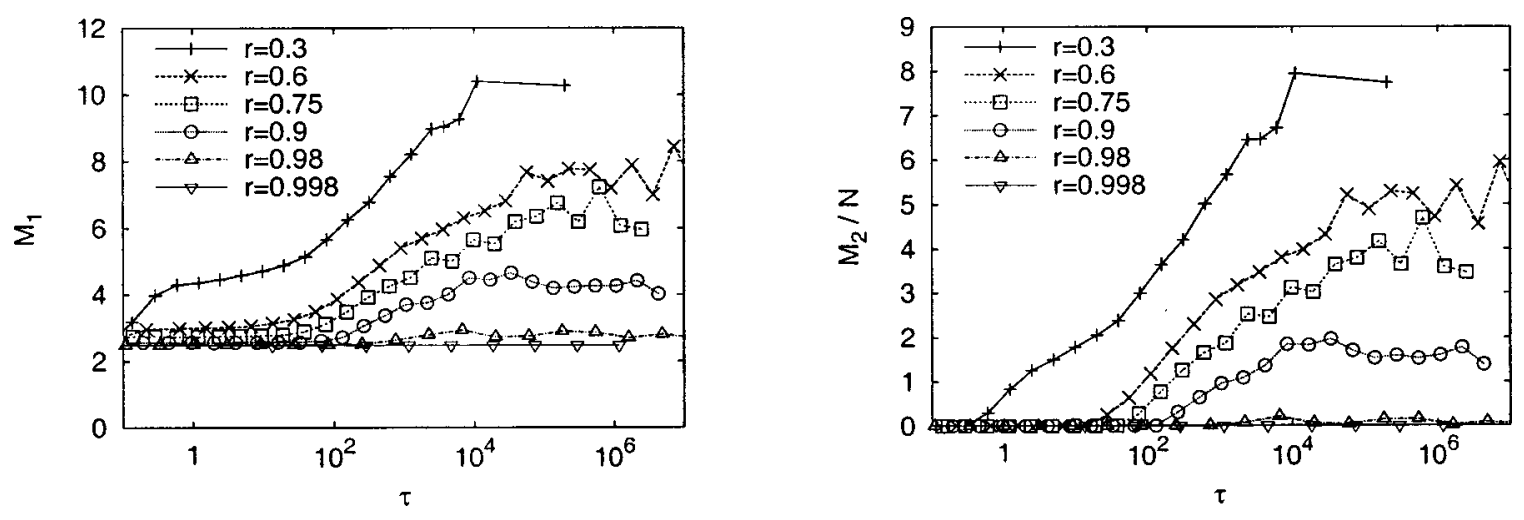

FIG. 5. Growth of the first moment $M_{1}$ (left) and the second moment $M_{2}$ (right) of the cluster size distribution plotted against scaled time $\tau$ in a 3D system with $N=512000$ particles, volume fraction $\rho=0.25$, and different restitution coefficients $r$ (increasing $r$ from top to bottom).

we use the following (geometrical) definition: Two particles belong to the same cluster if their distance is smaller than $s=0.1$ particle diameters. The choice of $s$ is arbitrary and shifts the results; the qualitative behavior of the quantities discussed below does not depend on $s$, as long as it is neither too small nor too large. ${ }^{1}$

The moments $M_{k}$ of the cluster size distribution are defined as

$$
M_{k}:=\frac{1}{n_{c}} \sum_{i} i^{k} n_{i}
$$

where $n_{c}$ denotes the total number of clusters and $n_{i}$ the number of clusters of size $i$.

In many cases there are a lot of small clusters and one large cluster of size $N_{x}$, which contains a macroscopic fraction $m_{x}:=N_{x} / N$ of the total number $N$ of particles. Therefore, we also define reduced moments $M_{k}^{\prime}$, which do not include the largest cluster.

In Figs. 4 and 5 the growth of the clusters can be seen on the basis of the first and second moments. After several collisions, particles start to cluster and the moments of the cluster size distribution grow until they reach their "saturation" value. $^{2}$ A numerical analysis reveals that the increase in $M_{1}$ and $M_{2}$ is mainly due to one large cluster which grows until it reaches its maximum size. In this final state this cluster contains a macroscopic fraction $m_{x}$ of the particles (see Fig. 6).

The onset time of cluster growth and also the final size $m_{x}$ of the large cluster depend strongly on the restitution coefficient $r$ (see Figs. 4 and 5). At low dissipation rates, for a long time nothing interesting happens and finally small and strongly fluctuating clusters appear. High dissipation leads to

\footnotetext{
${ }^{1}$ For a detailed study of different $s$ in 2D see Ref. [9]; in 3D, the $s$ dependence of some quantities seems to be stronger than for others.

${ }^{2}$ Saturation means that there is a transition to a regime with a much slower change of the moments. The dynamics is not finished, however, since the large cluster still can collect particles or break into pieces.
}

almost immediate cluster growth and a very large cluster at last. On the other hand, the system size $N$ does not seem to affect the behavior of the system provided that $N$ is not too small (see Fig. 6).

It has been shown $[6,8,21]$ that for infinite system size the system is always unstable to the formation of clusters, whereas for a finite system size the density $\rho$ and the dissipation $\lambda=1-r^{2}$ must not be too small. With our choice $N$ $\geqslant 10^{5}$ and $\rho=0.25$ we expect no cluster formation below the critical dissipation $\lambda_{c}=10^{-4}$ in our $2 \mathrm{D}$ system and $\lambda_{c}$ $=10^{-2}$ in our $3 \mathrm{D}$ system [8]. This is in good agreement with our results for the 3D system, where cluster formation still can be seen with dissipation $\lambda=0.04$, but not for $\lambda=0.004$ (see Fig. 5).

As can be seen in Fig. 7 (top) the onset $\tau_{c}$ of the growth of the large cluster in 2D does not depend on the restitution coefficient $r$ explicitly. (Implicitly, $\tau_{c}$ is dependent on $r$ via rescaled time, of course.) In contrast $\tau_{c}$ does strongly depend on $r$ in a 3D system [see Fig. 7 (bottom)].

An empirical formula, which gives the dependency rather accurately over orders of magnitude in $\lambda$, is

$$
\tau_{c}(\lambda)=\tau_{1}+c \frac{(1-\lambda)^{2}}{\lambda-\lambda_{c}}
$$

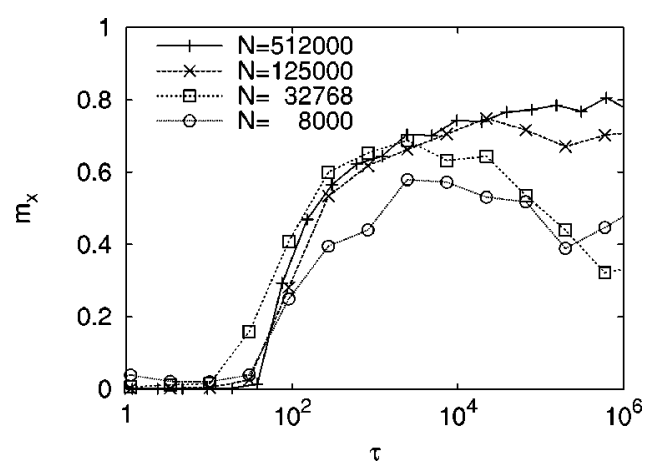

FIG. 6. Growth of the large cluster $m_{x}$ in a 3D system with $N$ particles, volume fraction $\rho=0.25$, and a restitution coefficient $r$ $=0.75$. 

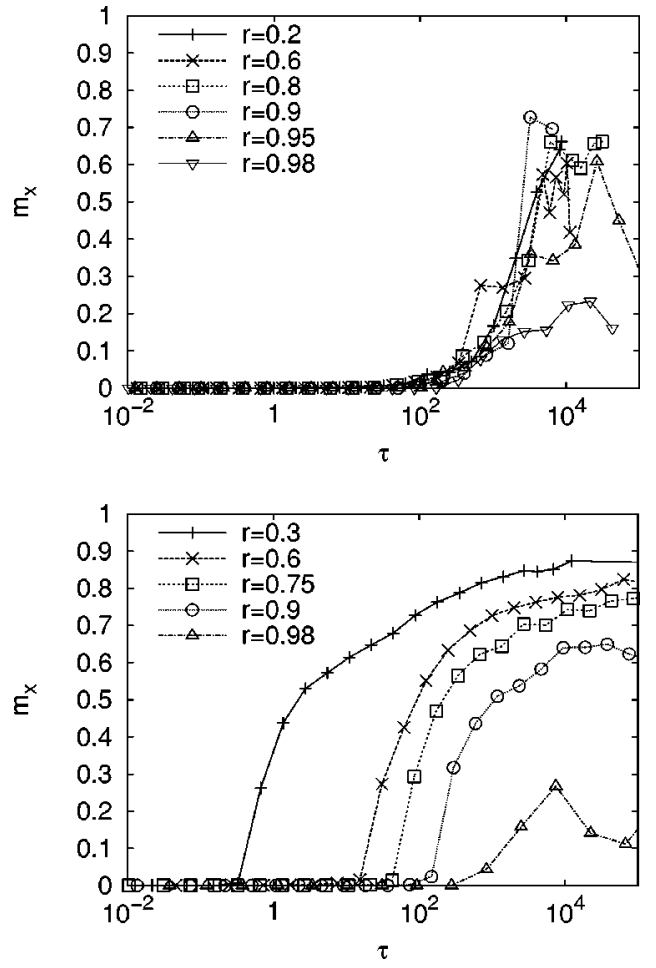

FIG. 7. Growth of the large cluster $m_{x}$ in 2D (top) and 3D (bottom) for different restitution coefficients $r$.

where $\tau_{1}=0.24 \pm 0.02$ and $c=36 \pm 5$ are fit parameters (for $s=0.1$ ).

In the limit $\lambda \rightarrow 1$, one has $\tau_{c} \rightarrow \tau_{1}$, where $\tau_{1}$ is proportional to the rescaled collision frequency and corresponds to $t=2 D \tau_{1} t_{E} / \lambda \approx 1.5 t_{E}$. This constant stems from the fact that the particles need at least one collision to cluster, however, it also depends on $s$. In the limit $\lambda \rightarrow \lambda_{c}$ the time $\tau_{c}$ diverges: the particles never start to cluster. ${ }^{3}$

Rescaling time in Fig. 7 (top) according to Eq. (6) results in Fig. 8 (bottom). There, the onset of the clustering happens approximately at the same rescaled time, whereas these times differ by four orders of magnitude in Fig. 7 (right). The fluctuations in $\tau_{c}$ are not systematically dependent on $r$.

Another difference between the 2D and 3D system in Fig. 7 is the fact that the cluster growth in 2D starts very smoothly, whereas the beginning of the clustering is very sharp in $3 \mathrm{D}$.

\section{Critical exponents in 3D}

As the moments of the cluster size distribution are clearly dominated by the large cluster, we will now study the reduced second moment $M_{2}^{\prime}$, which does not include the large cluster. Figure 9 shows that $M_{2}^{\prime}$ is small most of the time, except for a peak at $\tau_{c}$. This gives us a clean definition of $\tau_{c}$. But what is more interesting is that parallels to percola-

\footnotetext{
${ }^{3}$ Since we focused on strong dissipation in this study, rather than the limit $\lambda \rightarrow \lambda_{c}$, we cannot draw a conclusion on the functional behavior from our limited data.
}
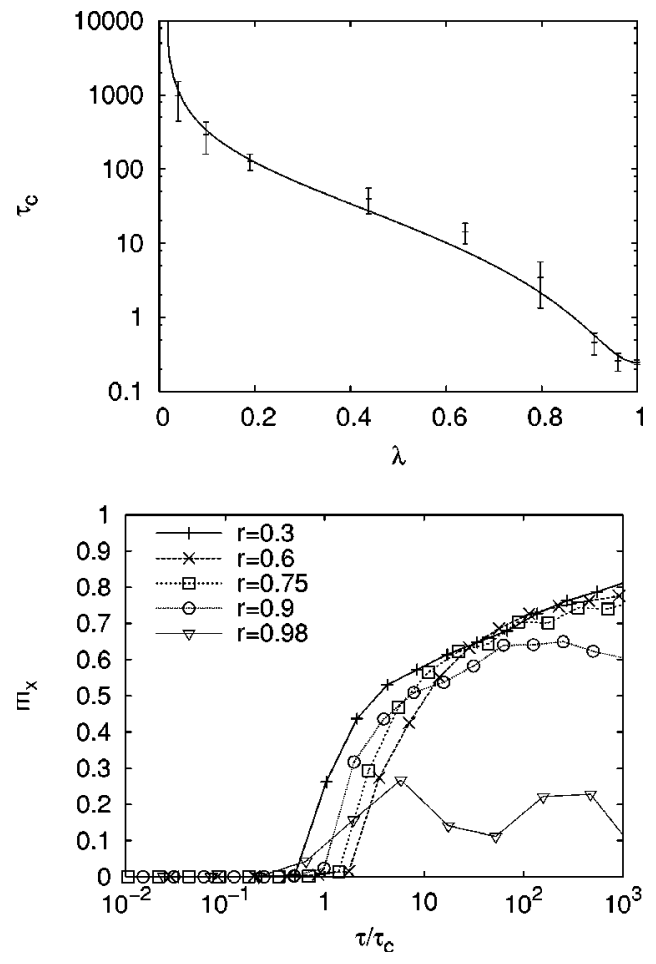

FIG. 8. (Top) Scaling behavior of the onset $\tau_{c}$ of the cluster growth in $3 \mathrm{D}$ depending on the dissipation rate $\lambda$. The curve is Eq. (6). (Bottom) With rescaled time $\tau / \tau_{c}$ according to Eq. (6) the curves of the growth of the large cluster in $3 \mathrm{D}$ almost collapse.

tion theory arise. In percolation theory [22] one studies the scaling behavior of certain quantities depending on the occupation probability $p$ around the percolation threshold $p_{c}$. One result for the reduced second moment $M_{2}^{\prime}(p)$ is, e.g.,

$$
M_{2}^{\prime}(p) \sim\left|p-p_{c}\right|^{-\gamma}
$$

where $\gamma$ is a universal critical exponent. In order to transfer this result to cluster growth, we replace $p$ with $\tau$ and $p_{c}$ with $\tau_{c}$ :

$$
M_{2}^{\prime}(\tau) \sim\left|\tau-\tau_{c}\right|^{-\gamma}
$$

Indeed we find in Fig. 9 the same power law behavior. All data for different system sizes collapse on the same master curve and even the exponent $\gamma=1.8 \pm 0.1$ is in agreement with the 3D percolation problem result $\gamma=1.80$ after the beginning of clustering.

Now, if we study the amplitude of this peak, percolation theory tells us that the maximum sits at

$$
p_{\max } \sim p_{c}\left(1-a L^{-1 / \nu}\right)
$$

where $L$ is the system size, $a$ is a constant, and $\nu$ is another critical exponent. Thus we expect

$$
M_{2}^{\prime}\left(p_{\max }\right) \sim L^{\gamma / \nu}
$$



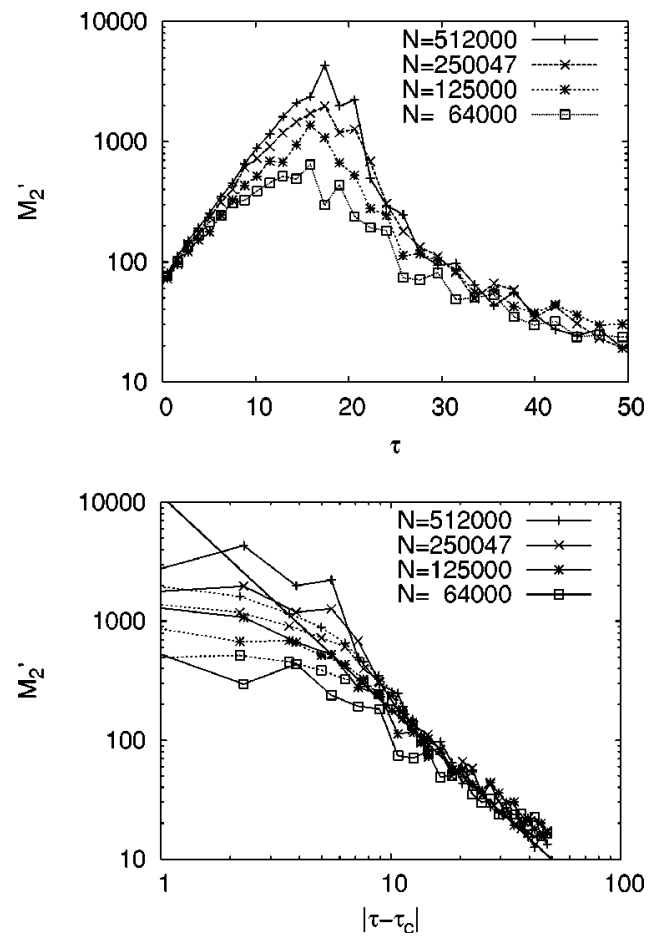

FIG. 9. Reduced second moment $M_{2}^{\prime}$ of the cluster size distribution in 3D systems with different $N$, volume fraction $\rho=0.25$, and a restitution coefficient $r=0.6$. The data are averaged over 10-20 different simulation runs. (Top) The curves show a peak at the onset $\tau_{c}$ of the cluster growth. (Bottom) Scaling behavior of $M_{2}^{\prime}(\tau)$ against $\left|\tau-\tau_{c}\right|$; each data curve has two branches, solid and dotted lines connect data points for $\tau>\tau_{c}$ and $\tau<\tau_{c}$, respectively. The additional line has a slope of -1.8 .

As the system size $L \sim N^{1 / 3}$, the maximum value of the peak in Fig. 9 scales as

$$
M_{2, \max }^{\prime}(N) \sim N^{\gamma / 3 \nu}
$$

Here we can also verify the power law behavior (see Fig. 10). Our simulations yield the result $\gamma / 3 \nu=0.77 \pm 0.09$, which leads to $\nu=0.78 \pm 0.14$. Within the rather large margin of errors this result is close to the $3 \mathrm{D}$ percolation problem $\nu=0.88$, too.

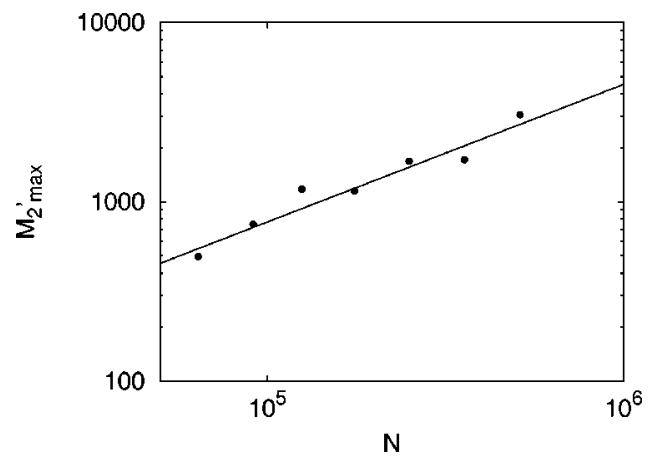

FIG. 10. Maximal amplitude of the reduced second moment $M_{2}^{\prime}$ of the cluster size distribution (see Fig. 9). The line has a slope of 0.77 .

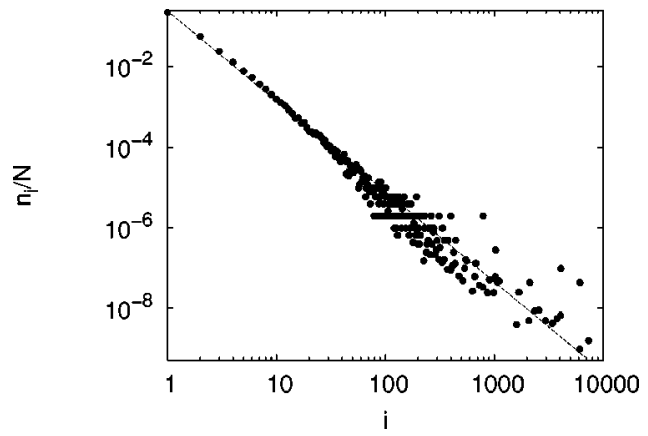

FIG. 11. The points give the numbers $n_{i}$ of clusters of size $i$ at time $\tau_{c}$ in a $3 \mathrm{D}$ simulation with $N=512000$ particles, volume fraction $\rho=0.25$, and a restitution coefficient $r=0.75$. The line has a slope of -2.2 .

A third exponent ${ }^{4} q$ is given by the cluster size population at time $\tau_{c}$ :

$$
n_{i}\left(\tau_{c}\right) \sim i^{-q}
$$

where $n_{i}$ is the number of clusters of size $i$.

Figure 11 yields $q=2.2 \pm 0.2$. This result is in agreement with the prediction of percolation theory $q=2.2$, too.

Is this similarity of clustering in $3 \mathrm{D}$ and percolation coincidence or is there a deeper reason?

\section{Consequences}

Percolation theory makes many universal predictions for randomly disordered systems. The particles in a granular gas form such a disordered system. There have been other attempts to apply percolation theory to granular systems [23], and also more advanced phase ordering models have been used to parallel the clustering dynamics $[11,12]$. Our results indicate that such attempts can be justified, even though the percolation problem is purely static, whereas the clustering of granular gases also involves the dynamics, i.e., momentum and energy.

The only thing that might seem strange at first sight is the derivation of Eq. (8). Why can we replace the purely static quantity $p$ with time $\tau$ ?

In order to introduce the occupation probability $p$ in the clustering problem, we define it as

$$
p(\tau):=\frac{M_{1}(\tau)-M_{1}\left(\tau^{*}\right)}{M_{1}(\tau)},
$$

where $\tau^{*} \ll \tau_{c}$ is a time shortly after the first few collisions. Now, we examine $p(\tau)$ around $\tau_{c}$.

Figure 12 (right) shows that in $3 \mathrm{D}, p$ is almost proportional to $\tau$. If we make use of this linear relation and insert it in Eq. (7), we arrive at the postulated formula, Eq. (8).

In contrast, in our 2D system the growth of the large

\footnotetext{
${ }^{4}$ The common notation for this exponent is $\tau$. In order to avoid name conflicts we call it $q$.
} 

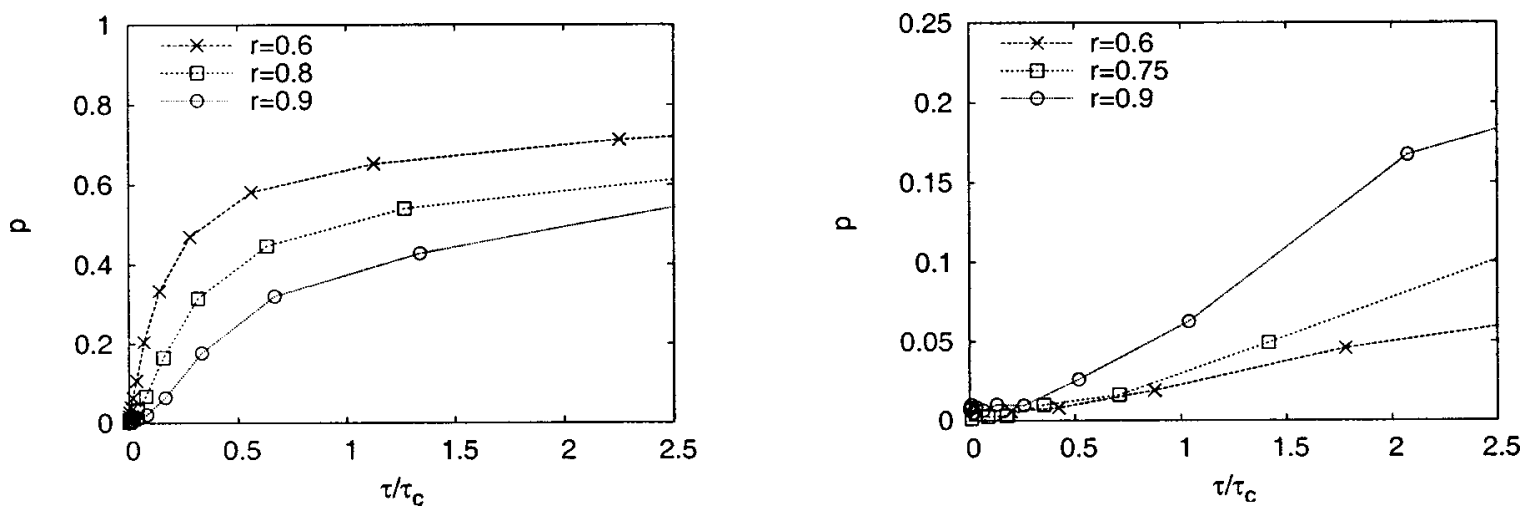

FIG. 12. "Occupation probability" $p$ against scaled time $\tau / \tau_{c}$. (Left) In $2 \mathrm{D}$ the data curves are highly nonlinear. (Right) In 3D the data curves are almost linear.

cluster happens at a rather large time $\tau_{c}$. (Note that $\tau_{c}$ is not very clearly defined in the $2 \mathrm{D}$ case, because the onset of the cluster growth is very smooth.) In this late stage, the relation between $p$ and $\tau$ is highly nonlinear, see Fig. 12 (left). Thus we could not find universal critical exponents for $\tau$ there. However, it might be possible to find a linear relation for a different set of parameters, e.g., for a different volume fraction, too. Whether a 2D system and a 3D system with the same parameters (such as, e.g., volume fraction) are in fact comparable is another open issue.

\section{SUMMARY AND DISCUSSION}

The evolution of freely cooling granular systems can be divided into three regimes. First, the system is in the HCS. Then, in the cluster-growth regime, clusters begin to develop and grow. Finally, in the saturation regime, the clusters merge to practically one large cluster, which grows until it reaches system size. Besides the macroscopic fraction of particles in the large cluster, there are still many small clusters with interesting statistics.

In the HCS, the decay of the kinetic energy $E$ and the collision frequency $f_{c}$ can be described by the simple analytical expressions $E(\tau) \sim(1+\tau)^{-2}$ and $f_{c}(\tau) \sim(1+\tau)^{-1}$. The collision frequency is the natural time scale here, mainly determined by the density and the dissipation rate of the system. For strong dissipation, at short range, particles already stay closer together after only one collision, so that the moments of the size distribution change rather early due to a (short-range) change in the radial pair distribution (data not shown).

In the cluster-growth regime, the collision frequency shows large fluctuations because of cluster-cluster collisions and cannot be predicted during cluster growth, because it changes erratically and discontinuously. The energy decay is characterized by $E \sim \tau^{-1}$, where the accuracy of the exponent is limited in $3 \mathrm{D}$ due to the comparatively short duration of the cluster-growth regime. However, this regime shows interesting differences between two and three dimensions. In $3 \mathrm{D}$, cluster growth can be described by a power law behavior with the same critical exponents as in percolation theory. The onset of this cluster growth $\tau_{c}$ is very sharp and does strongly depend on dissipation $\lambda$. In contrast, we could not find a similar behavior in $2 \mathrm{D}$, where the onset of the cluster growth is very smooth and depends on dissipation $\lambda$ only implicitly.

When cluster growth has reached a dynamic equilibrium in the saturation regime, the system is dominated by one large cluster which contains a macroscopic fraction of the system. Note that this regime still has an interesting dynamics and smaller clusters interact with the large cluster. Neither the small clusters nor the large one are static and the latter loses or eats up particles or smaller clusters. Kinetic energy and collision frequency still fluctuate, but are governed by the equations $E(\tau) \sim \tau^{-2}$ and $f_{c}(\tau) \sim \tau^{-1}$. This means the evolution in time is similar to the homogeneous cooling state.

A very interesting observation is the similarity of clustering in 3D and percolation with respect to the critical exponents. Even for the dynamics of cluster growth we have found the same exponents as for the occupation probability in percolation theory. We have provided a tentative explanation which is based on the linear relation of the occupation probability $p$ and time $\tau$ around the onset $\tau_{c}$ of cluster growth in 3D. In 2D we did not find a power law behavior because the growth of the large cluster happens when the linear relation between $p$ and $\tau$ has disappeared-at least for the given set of parameters.

However, there still remain some open questions: Why does $\tau_{c}$ depend on $r$ in 3D, but not in 2D? Why does the growth of the large cluster proceed smoothly in 2D and rather sharply in 3D? And why are all these differences present while the energy decay is proportional to $\tau^{-1}$ in both cases? Detailed studies of these questions and also of the cluster size probability distribution are in progress as well as a more systematic study of the cluster definition in 3D.

\section{ACKNOWLEDGMENTS}

This research was funded by the Deutsche Forschungsgemeinschaft (DFG) within the special research groups SFB 382 and SFB 404, and Grant No. LU/450/9-1. We thank Hans Herrmann and Sean McNamara for helpful discussions. 
[1] Physics of Dry Granular Media, Vol. 350 of NATO Advanced Studies Institute, Series E: Applied Sciences, edited by H. J. Herrmann, J.-P. Hovi, and S. Luding (Kluwer Academic, Dordrecht, 1998).

[2] Granular Gases, Lecture Notes in Physics Vol. 564, edited by T. Pöschel and S. Luding, (Springer, Berlin, 2001).

[3] Continuous and Discontinuous Modelling of Cohesive Frictional Materials, edited by P.A. Vermeer, S. Diebels, W. Ehlers, H.J. Herrmann, S. Luding, and E. Ramm, Lecture Notes in Physics Vol. 568 (Springer, Berlin, 2001).

[4] Powders \& Grains 2001, edited by Y. Kishino (Balkema, Rotterdam, 2001).

[5] R. Cafiero, S. Luding, and H.J. Herrmann, Phys. Rev. Lett. 84, 6014 (2000).

[6] I. Goldhirsch and G. Zanetti, Phys. Rev. Lett. 70, 1619 (1993).

[7] I. Goldhirsch, M.-L. Tan, and G. Zanetti, J. Sci. Comput. 8, 1 (1993).

[8] S. McNamara and W.R. Young, Phys. Rev. E 53, 5089 (1996).

[9] S. Luding and H.J. Herrmann, Chaos 9, 673 (1999).

[10] X.B. Nie, E. Ben-Naim, and S.Y. Chen, Phys. Rev. Lett. 89,
204301 (2002).

[11] S.K. Das and S. Puri, Physica A 318, 55 (2003).

[12] S.K. Das and S. Puri, Europhys. Lett. 61, 749 (2003).

[13] S. Luding, in Physics of Dry Granular Media, Vol. 350 of Advanced Studies Institute, Series E: Applied Sciences, edited by H. J. Herrmann, J.-P. Hovi, and S. Luding (Kluwer Academic, Dordrecht, 1998), p. 285, also see references therein.

[14] B.D. Lubachevsky, J. Comput. Phys. 94, 255 (1991).

[15] S. Miller and S. Luding, J. Comput. Phys. 193, 306 (2004).

[16] M.P. Allen and D.J. Tildesley, Computer Simulation of Liquids (Oxford University Press, Oxford, 1987).

[17] B.D. Lubachevsky, Int. J. Comput. Simul. 2, 373 (1992).

[18] S. Luding and S. McNamara, Granular Matter 1, 113 (1998).

[19] S. Luding, M. Huthmann, S. McNamara, and A. Zippelius, Phys. Rev. E 58, 3416 (1998).

[20] P.K. Haff, J. Fluid Mech. 134, 401 (1983).

[21] N. Sela and I. Goldhirsch, Phys. Fluids 7, 507 (1995).

[22] D. Stauffer and A. Aharony, Introduction to Percolation Theory, 2nd ed. (Taylor \& Francis, London, 1994).

[23] J. Tobochnik, Phys. Rev. E 60, 7137 (1999). 